\title{
STAR FORMATION HISTORIES OF SPIRAL GALAXIES
}

\section{FROM A MULTI-WAVELENGTH STUDY}

\author{
C. XU \\ Max-Planck-Institut für Kernphysik \\ Postfach 103980, D-69117 Heidelberg, Germany
}

We study the star formation histories of a sample of 113 nearby spiral galaxies using their radio continuum $(20 \mathrm{~cm}), \mathrm{FIR}(40-120 \mu \mathrm{m}), \mathrm{H}(1.65 \mu \mathrm{m})$ and $B(4400 \AA)$ luminosities. The first two are used as indicators of star formation rate over the past $\sim 10^{8}$ years, as suggested by the tight and nearly universal FIR/radio correlation ( $\mathrm{Xu}$ et al. 1994). Compared to other indicators of recent star formation rate such as $\mathrm{H} \alpha$ and UV, FIR and radio continuum have the advantage of being insensitive to extinction. The $B$ luminosity is taken as star formation indicator for the time scale of $310^{9}$ years, and the $\mathrm{H}$ luminosity for the time scale of $10^{10}$ years. We find:

1. The long-term star-formation history (from a few billion years to the entire Hubble time), as indicated by the B-to-H luminosity ratio, depends strongly on the Hubble type.

2. The recent star-formation history in the last a few billion years, as indicated by the radio-to-B luminosity ratio and the FIR-to-B luminosity ratio, does not depend on the Hubble type.

3. Galaxies of a given Hubble type have similar long-term star-formation histories. On the other hand, their recent star-formation histories in the last a few billion years can be much different: the ratio between the star-formation rate averaged over the past $10^{8}$ years to that over the past $310^{9}$ years can be different by two orders of magnitude, as indicated by the scattering of the radio-to-B luminosity ratio and that of the FIR-to-B luminosity ratio. This is not likely to be due to the extinction on the B luminosity, because the scattering of the B-to-H luminosity ratio for a given type is much smaller.

\section{References}

Xu, C., Lisenfeld, U., Völk, H.J., Wunderlich, E. 1994, A\&A 282, 19 